\title{
Safety of Cow's Milk-Derived Fortifiers used with an All Human Milk Base Diet in Very Low Birthweight Preterm Infants: Part II
}

Alan Lucas MD FMedSci, Maushumi Assad MD, MPH, Jan Sherman PhD, John Boscardin PhD, Steven Abrams MD

\begin{abstract}
Recently we published a meta-analyses of morbidity seen with the use of cow's milk derived fortifier (CMDF) rather than human milk derived fortifier (HMDF) in very low birthweight (VLBW) infants. Here, we further analyse these data to estimate the annual population risk of CMDF-related major morbidity in the United States and Canada. The outcome used was a mortality/morbidity index which was positive if the infants had one or more of death, necrotising enterocolitis, sepsis retinopathy of prematurity or bronchopulmonary dysplasia. Using the risk difference (RD) between the CMDF and HMDF groups we estimated, provisionally, that 4150 additional VLBW infants in the United States and Canada each year, or an additional infant approximately every 2 hours, may be expected to develop a positive mortality/morbidity index in relation to being fed CMDF - over and above the number of infants with a positive index if fed HMDF. We provide an in-depth discussion of the limitations of our estimate. This analysis provides preliminary evidence of the magnitude of population risk of major neonatal morbidity with use of CMDF versus HMDF in VLBW infants in current practice.
\end{abstract}

Key Words: preterm infant feeding, cow's milk-derived fortifiers, human milk-derived fortifiers, neonatal morbidity, neonatal mortality

Abbreviations: MOM: Mothers own milk, CM: Cow's milk, CMDF: Cow's milk-derived fortifier, PTF: Preterm fortifier, HMDF: human milk-derived fortifier, NEC: Necrotizing enterocolitis, ROP: Retinopathy of prematurity, BPD: bronchopulmonary dysplasia, NICU: Neonatal intensive care unit

\section{Introduction}

Recently we published our paper on "Safety of cow's milk-derived fortifiers used with an all-human milk base diet in very low birthweight preterm infants" (1). Here we seek to re-explore these data to quantify further the impact of cow's milk-derived fortifiers (CMDF) on morbidity in very low birthweight preterm infants in the United States and Canada, where the underlying studies were conducted.

Our recent paper (1) included a meta-analysis of 3 studies in 453 preterm infants all fed a base diet of human milk (HM) and comparing CMDF with human milk-derived fortifier (HMDF). Conventionally, we had expressed morbidity outcomes as a risk ratio $(\mathrm{RR})$. For example, the risk of developing necrotising enterocolitis (NEC) in the CMDF group was 3.3 times that in the HMDF group. However, expressing the morbidity data as a "risk difference" (RD) between the proportion of subjects that had adverse events in the CMDF versus HMDF groups has an important additional clinical application that we had not evaluated in the previous report. For instance, when multiplied by a population size of interest, the risk difference provides an estimate of the number of subjects in that population that might be adversely affected by CMDF over and above that number in the HMDF group. The reciprocal of the risk difference gives an estimate of the number needed to harm (NNH).

Here we have focussed on the risk difference for the most robust of the morbidity outcomes in our recent study: the meta-analysis of the mortality/morbidity index, which was positive if a subject had one or more of the following outcomes: Death, NEC, sepsis, retinopathy of prematurity (ROP), and bronchopulmonary dysplasia (BPD). Our objective has been to use the currently available data to define the number needed to harm and to derive an initial, provisional estimate of the number of VLBW preterm infants each year in the United States and Canada whose death or major neonatal morbidity may be linked to CMDF.

\section{"Our focus here on this overall index rather than on the individual morbidity outcomes reflected an attempt to use the most statistically robust outcome for our present analysis."}

\section{Methods}

The three studies in our meta-analysis in the original recent paper were: (1) the randomised controlled trial of O'Connor et al(2) (known as the OptiMoM trial) (2) a subgroup analyses of the RCT by Sullivan and co-workers (3.4) and (3) a subgroup analysis of a study by Assad and co-workers $(1,5)$ that provided a quasiexperimental pre-post design using $\mathrm{HM}$ and a CMDF pre-2012 and HM plus an HMDF post-2012. Our recent paper and the studies, as originally published, provide data on the methods used in each study(1-5). Within each individual study included in our meta-analysis, the CMDF and HMDF groups were well balanced for demographic factors(1); and in each of these three studies, the study design was conceptually similar: a base diet comprising only HM (either mother's own milk [MOM]; or MOM plus donor milk [DM]) and comparing a CMDF versus HMDF.

In each of the studies, the HMDF group received Prolact+ $\mathrm{H}(2)$ MF® human milk fortifier (manufactured by Prolacta Bioscience, California, USA) and the CMDF group received Enfamil or Similac

NEONATOLOGY TODAY is interested in publishing manuscripts from Neonatologists, Fellows, NNPs and those involved in caring for neonates on case studies, research results, hospital news, meeting announcements, and other pertinent topics.

Please submit your manuscript to: LomaLindaPublishingCompany@gmail.com 
non-hydrolyzed human milk fortifier (manufactured respectively by Mead Johnson Nutrition or Abbott Nutrition). Feeding protocols in each study are described elsewhere (1-5).

Morbidity outcomes studied here were death, NEC (Bell's stage II or greater), sepsis, ROP, and BPD. The criteria for the diagnosis of NEC and sepsis are described elsewhere (3). These five outcomes were combined in a dichotomous mortality/morbidity index, which was positive if one or more of these individual morbidities present. This index was first used in the O'Connor trial (2), and since the other two studies provided data on the same outcomes, a meta-analysis of this mortality/morbidity index was possible. Our focus here on this overall index rather than on the individual morbidity outcomes reflected an attempt to use the most statistically robust outcome for our present analysis.

\section{"When we re-analyzed the data for the mortality/morbidity index for the risk difference (RD), the chosen random- effects model provided a similar overall value for this as the fixed effect models (RD of 0.13 and 0.14 respectively) with similar significance."}

In our recent study(1), we justified, following the guidance of Borenstein (6), using a fixed-effect model in our meta-analysis of morbidity indices because of the paucity of studies in the analysis, but we noted that a random-effects model gave a similar result. Our fixed effect model effectively treated the three studies as the only ones of interest, but this was not the objective here for a model that should ideally be representative of all studies. Therefore, for this study, focusing on the mortality/morbidity index and using the same software (RevMan 5.3), we selected the now preferred random-effects model, using this to estimate "risk difference."

\section{Results}

When we re-analyzed the data for the mortality/morbidity index for the risk difference (RD), the chosen random-effects model provided a similar overall value for this as the fixed effect models (RD of 0.13 and 0.14 respectively) with similar significance. The findings for the random-effects model were as follows: risk difference $=0.14$ (95\% Cl: 0.05 to 0.23$) \mathrm{P}=0.003$ (see figure). The risk difference of 0.14 reflects the fractions of subjects with a positive index in the CMDF group minus the fraction in the HMDF group.

\section{Number needed to harm (NNH)}

The reciprocal of this risk difference (the estimated $\mathrm{NNH}$ ) was 7 (7.1), with a 95\% confidence interval of 4 to 25 . Thus, from the point estimate, for every seven babies fed on CMDF versus HMDF, one of them would be predicted to die or have one or more of the four severe morbidities.

\section{Provisional population estimates}

Given an estimated risk difference of 0.14 or $14 \%$ as an absolute percentage, then if our meta-analysis were to comprise studies that are collectively representative of the population (USA plus Canada in this study), this implies that $14 \%$ of preterm infants in that population is an estimate of the increased number of infants with a positive mortality/morbidity index in the CMDF group over and above the number with a positive index in the HMDF group.

For instance, the data for the OptiMoM trial by O'Connor et al. included in the figure shows that 30 of $64(0.49)$ of the CMDF group had a positive mortality/morbidity index and 23 of $64(0.36)$ had a positive index. Thus, the difference known as the "risk difference" (RD) is 0.13 , as shown in the figure. If O'Connor's results were applied to a relevant population of, for example, 1000 preterm infants, then $130(1000 \times 0.13)$ additional infants would be expected to have a positive index if fed CMDF as opposed to HMDF.

\section{Estimations for the USA.}

The 12-center Sullivan study(3) indicated that many centers may use $<1250 \mathrm{~g}$ as a cut-off value for using HMDF. However, babies up to $1500 \mathrm{~g}$ birth weight were given HMDF in the Assad study(5) It is difficult to estimate precisely the number of preterm babies in the USA that might be eligible for the use of HMDF, but it is that

CM HM Risk Difference

Risk Difference

Study or Subgroup Events Total Events Total Weight M-H, Random, $95 \% \mathrm{Cl}$

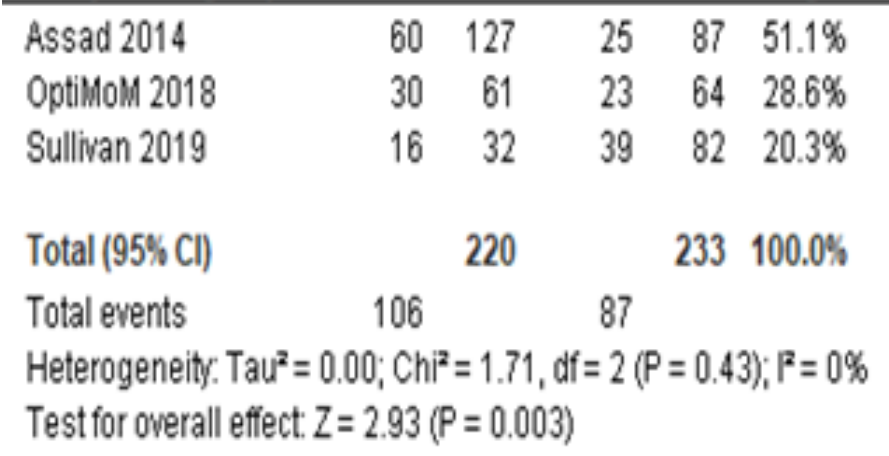

$0.19[0.06,0.31]$

$0.13+-0.04,0.30]$

$0.02[-0.18,0.23]$

$0.14[0.05,0.23]$ M.H, Random, $95 \% \mathrm{Cl}$

Figure: Meta-analysis of morbidity indices in the O'Connor, Assad, and Sullivan studies, A positive index is defined as one or more of death, sepsis, NEC, ROP and BPD. The index was based on the one published in the O'Connor corrigendum20, and equivalent indices (based on the same 5 outcomes) were derived from raw data in the Sullivan and Assad reanalysis. Note that the O'Connor study is also known as the 'OptiMoM' trial)

The meta-analysis is a random effects model for risk difference: CMDF v HMDF 
number that is required to estimate CMDF-related morbidity over and above that seen with HMDF. From the National Vital Statistics Report of Martin et al. (2018)(7), we note that all births in the United States approach 3.8 million/year and that $1.38 \%$ of these are below $1500 \mathrm{~g}$ at birth (VLBW). We have assumed that the eligible population for receiving HMDF is close to $1 \%$ of all births in the United States - about 38,000 preterm infants based on current live birth rates.

On this basis, a tentatively estimated $5300(0.14 \times 38,000)$ CMDFfed babies annually in the USA would have a positive mortality/ morbidity index over and above the incidence in the HMDF group but with a $95 \%$ confidence interval of 1900 to 8700 babies.

Nevertheless, $30 \%$ of this morbidity has already been addressed in the USA, where close to 11,000 VLBW babies are fed annually on HMDF to provide an exclusive HM (EHM) diet (data on the number of babies fed HMDF obtained by courtesy of the manufacturer). Our analysis thus provides a point estimate of 3,700 babies in the United States each year $(70 \%$ of 5300$)$ who may die or develop one of more major morbidities in relation to use of CMDF rather than HMDF.

\section{Estimations for Canada}

The annual number of births in Canada is approximately $373,000(8)$. Assuming again that the target population of preterm infants represents $1 \%$ of births, this would amount to 3730 infants. Our provisional estimate based on the risk difference of $0.14,522$ babies would have a positive mortality/morbidity index related to the use of CMDF over and above the number with a positive index if fed HMDF. However, in 2019, 75 Canadian preterm infants were fed on HMDF (manufacturer's data), leaving a point estimate of around 450 babies in Canada each year who may die or develop major morbidity in relation to the use of CMDF,

\section{"However, we were concerned that}

this analysis was not an accurate representation of the data. Our own analysis utilized the authors' data on individual morbidities to calculate the average number of adverse events per subject. There were 31 adverse events in 64 subjects fed HMDF $(0.48$ events per subject); yet, for CMDF there were 45 adverse events among 61 subjects $(0.74$ events per subject)."

\section{Combined data}

Combining the figures for the United States and Canada, an estimated total of $4150(95 \% \mathrm{Cl} 1480,6800)$ additional babies in the two countries would have a positive mortality/morbidity index related to the use of a CMDF rather than HMDF.

\section{Discussion}

Our provisional analysis for the USA and Canada indicates that with the use of CMDF rather than HMDF, an additional 4150 VLBW preterm infants per year - or on average one additional infant every 2 hours - would develop a positive mortality/morbidity index comprising one or more of the following major adverse outcomes: death, NEC, proven sepsis, ROP or BPD. Such raised morbidity rates in the population in those fed CMDF might not have been intuitively predicted from the risk ratio we reported in the original study that showed an estimated $40 \%$ increase in the risk of a positive index in the CMDF group.(1) In terms of number needed to harm (NNH), for every seven babies in our analysis that were fed CMDF rather than HMDF, one of them is estimated to develop one of more of these adverse outcomes. However, these preliminary and tentative figures are based on the few studies of suitable design available, and in this discussion, we examine both the plausibility and limitations of our estimates.

In terms of plausibility, decades of prior research have linked the feeding of cow's milk-derived products with the outcomes that comprise the mortality/morbidity index used here (1-3,5,9-16). Indeed, O'Connor and colleagues (2) conceived this index for their own fortifier trial, included in our meta-analysis, precisely because these were the major neonatal morbidities described with $\mathrm{CM}$ products in the past.

Over the past $7-8$ years, the recommended practice $(17,18)$ for VLBW preterm infants is to use DM if there is insufficient MOM; and then most commonly to use a CMDF. Given the preponderance of $\mathrm{HM}$ in the modern preterm infant diet, it might be questioned whether there was sufficient CM in the diet to cause so many cases of major morbidity. The preterm infant feeding trials of Lucas et al.(11) date back to the pre-fortifier era when babies were fed on $\mathrm{HM}$ and quite often a standard infant formula with much lower CM protein content than a preterm formula (PTF), yet NEC and sepsis were recognized CM-related problems at the time. Current use of CMDF may provide around $50 \%$ of the protein in the diet - and more than this if significant volumes of DM are used - comparable with the CM intake in past practice.

That CMDF may cause significant morbidity was also shown in an RCT on 276 subjects published in 1996 (19). Both randomized groups received MOM and PTF, but at that time, it was ethical to randomize the infants to a CMDF or not. With a quite modest level of fortification, simply adding a CMDF resulted in a 2.3-fold increased risk of NEC or sepsis

The analysis here raises a broader issue of how the results of meta-analyses may best be presented in different circumstances. The risk ratio (RR) provides a way of capturing the relative impact

NEONATOLOGY TODAY is interested in publishing manuscripts from Neonatologists, Fellows, NNPs and those involved in caring for neonates on case studies, research results, hospital news, meeting announcements, and other pertinent topics.

Please submit your manuscript to: LomaLindaPublishingCompany@gmail.com 
on how this relates to population incidence of, say, an adverse outcome using one of the interventions rather than the other and for this, the risk difference (RD) is a most valuable measure. However, extrapolating from limited data to population health may result in more speculative conclusions as noted in the Limitations and Conclusions sections below.

\section{Limitations}

Our recently published meta-analysis only had three studies that represented the data available to compare fortifier type and hence to explore the safety of current feeding recommendations in terms of mortality or major morbidity. We recognize that as more studies of this type are done, our estimate of the number of preterm VLBW that may be adversely impacted by CMDF may be refined, and the large confidence intervals we are currently seeing may contract.

Although we have used our meta-analytic data to estimate CMDFrelated morbidity and mortality both in the United States and Canada, only one of our three studies, the OptiMoM trial of O'Connor et al.(2), was from Canada where the practice may be different. However, the risk difference in this trial alone for the morbidity in$\operatorname{dex}(R D=0.13)$ was very similar to the value for the 3 study metaanalysis as a whole (RD=0.14).

"Our preliminary estimates presented here suggest that although about $30 \%$ of smaller VLBW babies in the USA are fed with HMDF, more than 4000 VLBW preterm infants in the United States and Canada each year may either die or develop major morbidity in relation to the use of CMDF."

The estimated magnitude of the adverse impact of CMDF could have been inflated by the inclusion of milder cases of ROP in the Assad study, though by studying five outcomes collectively in our mortality/morbidity index and having only one of the three centers collecting data on milder ROP cases, such confounding was minimized. In contrast, we have assumed that in modern care, DM would be used in preference to PTF (as in our three studies here); but that is not always the case, and because in those babies who receive both CMDF and $\mathrm{PTF}$, the adverse impact of $\mathrm{CM}$ may be somewhat increased, our model may have under-estimated the impact of CMDF. Again, in the Assad study(5), the largest of our studies, bigger babies (up to $1500 \mathrm{~g}$ birth weight) than those most commonly fed HMDF, were included - and because of the lower morbidity in larger preterm infants, this also would be expected generate a more conservative population estimate of the adverse impact of CMDF. It might be argued that as more modern studies are included, CMDF-related morbidity may decrease. This is not supported by the limited data we have, which indicates that the oldest study data we included (from the Sullivan trial published in 2010 (3) showed the least impact of CMDF on the mortality/ morbidity index with a much smaller impact on this index than the most recent study of O'Connor published in 2018 (2). This could relate to a secular trend to increasing accuracy in defining the morbidities studied here.

\section{Conclusions}

Our preliminary estimates presented here suggest that although about $30 \%$ of smaller VLBW babies in the USA are fed with HMDF, more than 4000 VLBW preterm infants in the United States and Canada each year may either die or develop major morbidity in relation to the use of CMDF. The estimated $\mathrm{NNH}$ of only 7 is also a concern. However, these estimates have a broad confidence interval and are based on the surprisingly small number of safety studies of this type. Our provisional analysis has several limitations, and we recognize it is difficult to extrapolate from small studies to large populations. Nevertheless, this field has become important to pursue - and these types of estimation will also need to be done on other populations, where the recommended neonatal feeding practices are similar.

\section{"As data have emerged, it has become clearer that these safety aspects are important to address and quantify. For this reason, we have released these data at an early stage to encourage both further research and discussion on the implications for practice."}

Our study illustrates a larger issue. Because of the broad adverse and beneficial impacts of preterm infant feeding on morbidity, feeding regimes in this sensitive period are helpfully seen as therapeutic interventions where both efficacy and safety are, in general, important metrics. The current recommendations for preterm infant feeding - notably to use DM if MOM is insufficient - were introduced over 20 years ago and have become globally common in the last ten years in part based on key position papers of relevant societies. $(17,18)$ Yet, at the time this guidance was given, there was an absence of randomized trials appropriately designed to address safety in terms of the morbidities examined here, when using CMDF as the sole source of CM - an integral component of this new practice. As data have emerged, it has become clearer that these safety aspects are important to address and quantify. For this reason, we have released these data at an early stage to encourage both further research and discussion on the implications for practice.

\section{Acknowledgments}

We acknowledge the kind permission of Dr Melinda Elliott for allowing us to reanalyse her raw data for the study by Assad et al. and for retrieving further raw data from the patient records.

\section{References}

1. Lucas, A., Assad, M., Sherman, J., Boscardin, J., \& Abrams, S. (2020). Safety of Cow's Milk-Derived Fortifiers used with an All-Human Milk Base Diet in Very Low Birthweight Preterm Infants. Neonatology Today. July, 15 (7), 3 - 13. http:// www.neonatologytoday.net/newsletters/nt-jul20.pdf

2. O'Connor D, Kiss A, Tomlinson $C$ et al. Nutrient enrichment of human milk with human and bovine milk-based fortifiers for infants born weighing <1250 g: a randomized clinical trial. Am J Clin Nutr. 2018. doi:10.1093/ajcn/nqy067

3. Sullivan S, Schanler R, Kim J et al. An Exclusively Human Milk-Based Diet Is Associated with a Lower Rate of Necrotizing Enterocolitis than a Diet of Human Milk and Bovine Milk-Based Products. J Pediatr. 2010;156(4):562-567.e1. doi:10.1016/j.jpeds.2009.10.040

4. Lucas A. Boscardin J, Abrams SA.Preterm infants fed cows' 
milk-derived fortifier had averse outcomes despite a base diet of only mother's own milk. Breast Feeding Medicine, 2020; 15(5): 1-7. DOI 10.1089/bfm.2019.0133

5. Assad M, Elliott M, Abraham J. Decreased cost and improved feeding tolerance in VLBW infants fed an exclusive human milk diet. Journal of Perinatology. 2015;36(3):216220. doi:10.1038/jp.2015.168

6. Borenstein, M. Hedges, L., \& Rothstein, H. (2007). MetaAnalysis: Fixed effect vs. random effects. (page 30) Retrieved from www.Meta-Analysis.com

7. Martin, J. A., Hamilton, B. E., Osterman, M., \& Driscoll, A. K. (2019). Births: Final Data for 2018. National vital statistics reports : from the Centers for Disease Control and Prevention, National Center for Health Statistics, National Vital Statistics System, 68(13), 1-47.

8. Statistics Canada (2020). Live births by month, 2018 to 2019. Retrieved from https://www150.statcan.gc.ca/t1/tb/1/ en/tv. action? pid=1310041501\&pickMembers $\% 5 B 0 \% 5 D=3$. 1 1\&cubeTimeFrame.startYear $=2018$ \&cubeTimeFrame.endY ear $=2019 \&$ referencePeriods $=20180101 \% 2$ C20190101

9. Cristofalo E, Schanler R, Blanco $C$ et al. Randomized Trial of Exclusive Human Milk versus Preterm Formula Diets in Extremely Premature Infants. J Pediatr. 2013;163(6):15921595.e1. doi:10.1016/j.jpeds.2013.07.011

10. Quigley M, Embleton N, McGuire W. Formula versus donor breast milk for feeding preterm or low birth weight infants. Cochrane Database of Systematic Reviews. 2018. doi:10.1002/14651858.cd002971.pub4

11. Lucas A, Cole T. Breast milk and neonatal necrotising enterocolitis. The Lancet. 1990;336(8730-8731):1519-1523. doi:10.1016/0140-6736(90)93304-8

12. Narayanan I, Prakash K, Murthy NS, Gujral VV. Randomised controlled trial of effect of raw and holder pasteurised human milk and of formula supplements on incidence of neonatal infection. Lancet 1984;ii:1111-3

13. de Silva $A$. Does human milk reduce infection rates in preterm infants? A systematic review. Archives of Disease in Childhood - Fetal and Neonatal Edition. 2004;89(6):F509F513. doi:10.1136/adc.2003.045682

14. Hair A, Rechtman D, Lee M, Niklas V. Beyond Necrotizing Enterocolitis: Other Clinical Advantages of an Exclusive Human Milk Diet. Breastfeeding Medicine. 2018;13(6):408-411. doi:10.1089/bfm.2017.0192

15. Abrams S, Schanler R, Lee M, Rechtman D. Greater Mortality and Morbidity in Extremely Preterm Infants Fed a Diet Containing Cow Milk Protein Products. Breastfeeding Medicine. 2014;9(6):281-285. doi:10.1089/bfm.2014.0024

16. Manzoni P, Stolfi $I$, Pedicino $R$ et al. Human milk feeding prevents retinopathy of prematurity $(R O P)$ in preterm VLBW neonates. Early Hum Dev. 2013;89:S64-S68. doi:10.1016/ s0378-3782(13)70019-7

17. AAP Section on Breastfeeding. Breastfeeding and the Use of Human Milk. Pediatrics. 2012;129(3):e827-e841. doi:10.1542/peds.2011-3552

18. ESPGHAN Committee on Nutrition, Arslanoglu S, Corpeleijn $W$, Moro $G$ et al. Donor human milk for preterm infants: current evidence and research directions. J Pediatr Gastroenterol Nutr. 2013;57(4):535-542. doi:10.1097/ mpg.0b013e3182a3af0a

19. Lucas $A$, et al. (1996). Randomized outcome trial of human milk fortification and developmental outcome in preterm infants. American Journal of Clinical Nutrition. August 1996. 64(2):142-51.

20. Corrigendum for O'Connor et al. Nutrient enrichment of human milk with human and bovine milk-based fortifiers for infants born weighing < $1250 \mathrm{~g}$ : a randomized clinical trial. Am J Clin Nutr 2018;108:108-16. Am J Clin Nutr. 2019;110:529.
Acknowledgments

We acknowledge the kind permission of Dr. Melinda Elliott for allowing us to reanalyze her raw data for the study by Assad et al. and for painstakingly retrieving further raw data from the patient records

\section{Author Contributions}

Professor Alan Lucas conceived and initiated the study; analyzed raw data; and wrote the paper

Dr Maushumi Assad provided her raw data (Assad study) and assisted us with the understanding of her database, She read and commented constructively on the manuscript

Professor Jan Sherman was our advisor on evidence-based medicine. She performed all the meta-analyses and provided the data for figures. She read and advised on the manuscript.

Dr. John Boscardin was our statistician who advised on: the statistical analysis, interpretation of data and data presentation

Professor Steven Abrams key collaborator involved in every aspect of the study and made major intellectual input.

Financial support: None:

Conflicts of Interest: Dr. Lucas has provided independent scientific advice to Philips, Prolacta, and Nestle.; Dr. Assad, none; Dr. Sherman, none; Dr. Boscardin, none. Dr. Abrams, none

\section{NT}
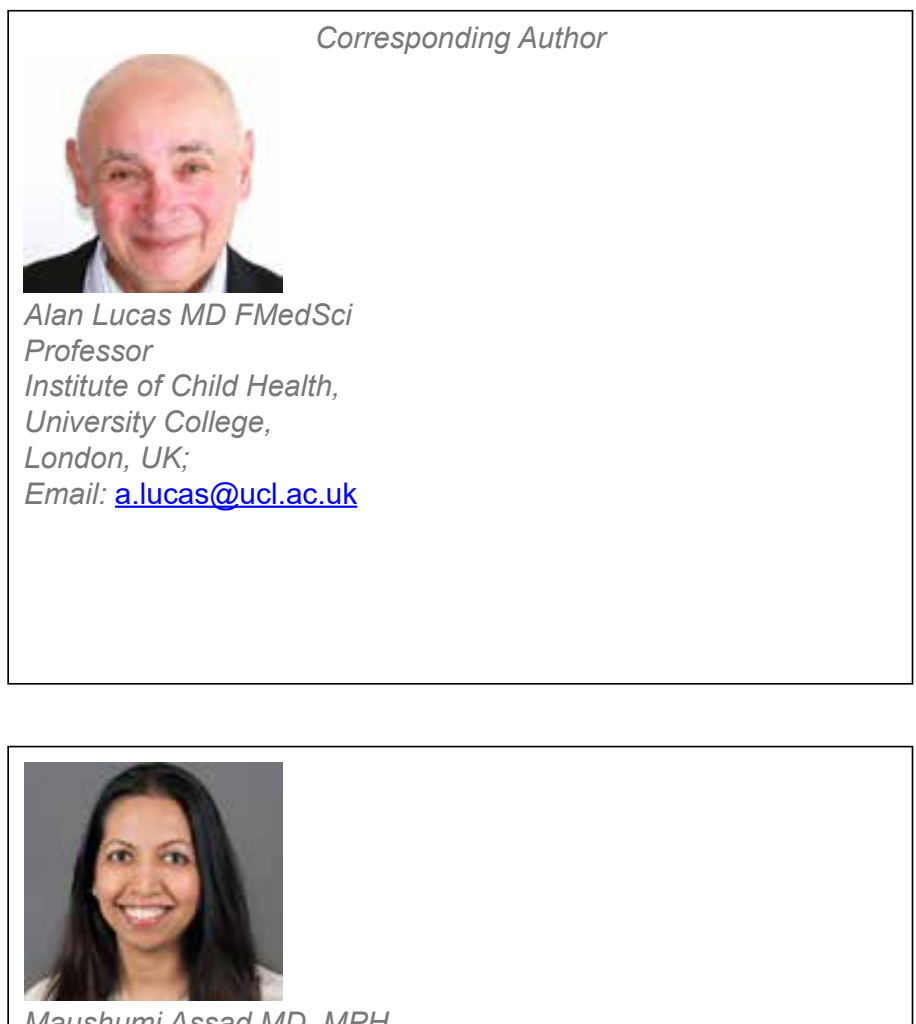

Maushumi Assad MD, MPH

Beth Israel Deaconess Medical Center,

Department of Neonatology,

Boston, MA, USA:

email: massad@bidmc.harvard.edu 


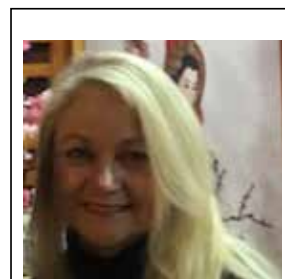

Jan Sherman PhD

Teaching Professor

School of Nursing

University of Missouri

Columbia, MO, USA

Adjunct Teaching Professor

School of Medicine

email: shermanj@missouri.edu

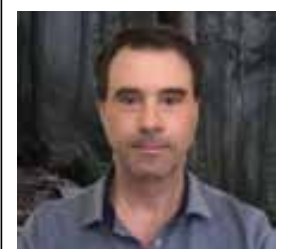

John Boscardin PhD

Professor

University of California

San Francisco, CA, USA

email: john.boscardin@gmail.com

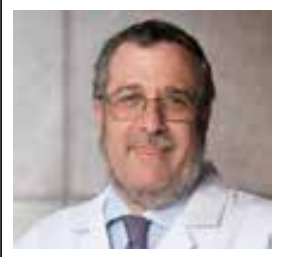

Steven Abrams MD

\section{Professor}

Department of Pediatrics

Dell Medical School

University of Texas,

Austin, TX, USA

email: sabrams@austin.utexas.edu
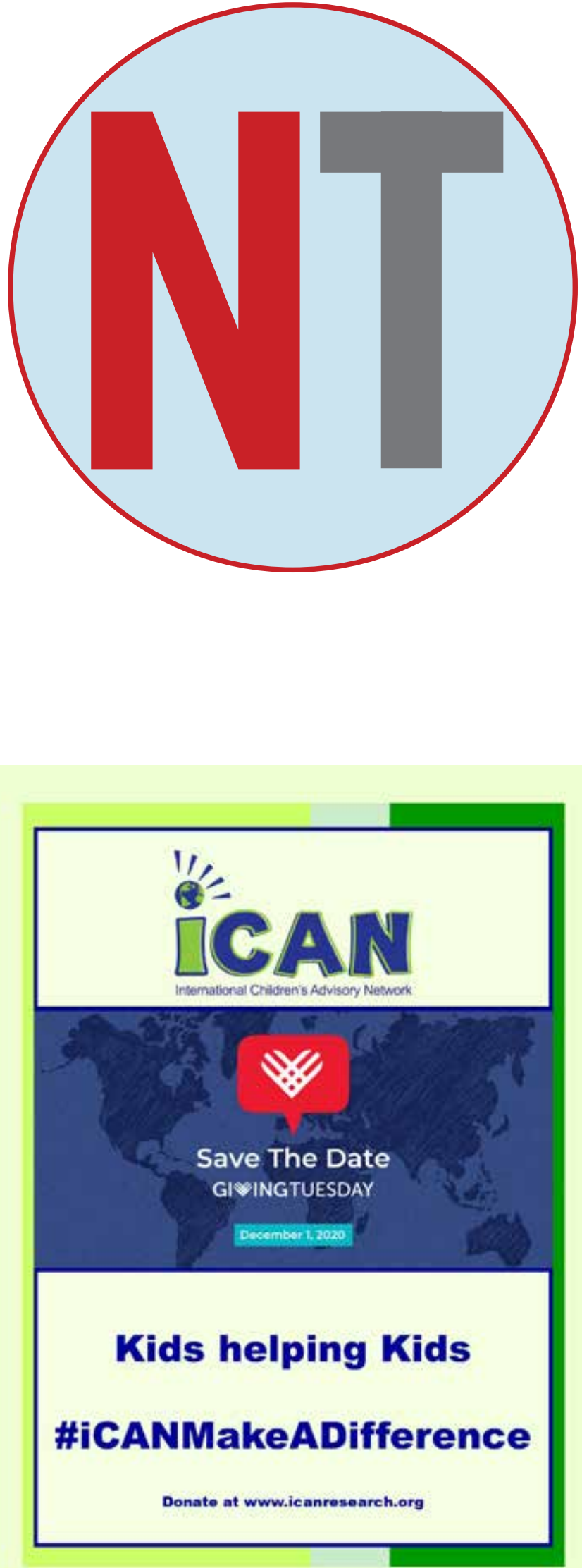